\title{
The Effects of Fish Oil Supplementation in Pregnancy on Breast Milk Fatty Acid Composition Over the Course of Lactation: A Randomized Controlled Trial
}

\author{
JANET A. DUNSTAN, LEON R. MITOULAS, GLENYS DIXON, DOROTA A. DOHERTY, PETER E. HARTMANN, \\ KAREN SIMMER, AND SUSAN L. PRESCOTT
}

\author{
School of Paediatrics and Child Health [J.A.D., G.D., K.S., S.L.P.], Biochemistry and Molecular Biology [L.R.M., P.E.H.], School of \\ Women's and Infants Health [D.A.D., K.S.], The University of Western Australia, Crawley WA 6009
}

\begin{abstract}
This study evaluated the longitudinal effect of fish oil in pregnancy on breast milk fatty acid composition and infant outcomes. In a randomized, controlled trial, 98 women received $2.2 \mathrm{~g}$ docosahexaenoic acid (DHA) and $1.1 \mathrm{~g}$ eicosapentaenoic acid (EPA) or olive oil from $20 \mathrm{wk}$ of gestation until delivery. Fatty acid composition in breast milk (at $3 \mathrm{~d}, 6 \mathrm{wk}$, and $6 \mathrm{mo}$ ) and infant erythrocyte membranes (at 1 y) were determined by gas liquid chromatography. Breast milk fatty acids were examined in relationship to growth and development. Compared with control group, breast milk from women who received fish oil had proportionally higher DHA and EPA levels at $3 \mathrm{~d}$ and 6 wk after delivery, but this difference was no longer apparent by 6 mo. Infant DHA status at $1 \mathrm{y}$ of age was directly related to DHA levels at $3 \mathrm{~d}, 6 \mathrm{wk}$, and 6 mo postpartum (but not to antenatal supplementation). Both EPA and DHA in breast milk were positively correlated with Griffith's developmental scores including hand and eye coordination. Thus, supplementation in pregnancy was associated with increased n-3 long-chain polyunsaturated fatty acids (LCPUFAs) in breast milk, particularly in early lactation, and this was positively associated with infant DHA status at 1 y. (Pediatr Res 62: 689-694, 2007)
\end{abstract}

$\mathrm{L}$ ong-chain polyunsaturated fatty acids (LCPUFAs_ are critical for optimal health and development. However, women in Western countries have much lower median n-3 LCPUFA intakes (around $30 \mathrm{mg} / \mathrm{d}$ in some studies) (1) compared with women from fish-eating nations such as Japan, Korea, and Norway where dietary n-3 LCPUFA intakes are $>1000 \mathrm{mg} / \mathrm{d}$. Dietary changes have been associated with changing composition of breast milk, which has shown decreasing n-3 LCPUFA content and increasing n-6 LCPUFA levels over a 20-y period (2). Furthermore, recent data indicate that pregnant women are actually likely to reduce their intake of fish in pregnancy in response to government advisories regarding consumption of specific fish that may contain high levels of methyl mercury (3). These observations have lead to more studies to determine the role of maternal fish oil supplementation.

Received May 9, 2007; accepted July 15, 2007.

Correspondence: Susan Prescott, M.D., Ph.D, School of Paediatrics and Child Health, PO Box D184, University of Western Australia, Perth, Princess Margaret Hospital, Perth WA 6001, Western Australia, Australia; e-mail: sprescott@meddent.uwa.edu.au

This study was partly funded by Medela AG. Janet Dunstan is funded by a fellowship from the Child Health Research Foundation of Western Australia.
Earlier studies of fish oil supplementation focused on supplementation of infants or postnatal supplementation of the mother to increase breast milk n-3 LCPUFA levels. Some studies suggest that improved LCPUFA status can improve functional outcomes.

We previously investigated the role of fish oil supplementation during pregnancy on infant immune function (4) and development (5). It was therefore logical to extend this study to explore the potential benefits of supplementation in pregnancy on subsequent breast milk composition as a continued source of n-3 LCPUFAs for infants. Since maternal diet in pregnancy has a marked impact on fatty acid status in the newborn, we hypothesized that maternal dietary supplementation with fish oil during pregnancy would also enhance maternal n-3 LCPUFA stores and hence breast milk levels and further contribute to subsequent n-3 LCPUFA status in the infant at $1 \mathrm{y}$ of age. Most previous studies focused on the acute effects of supplementation of the mothers' diet during lactation (6). To our knowledge, this is the first study to address the longitudinal effects of antenatal supplementation on breast milk n-3 LCPUFA status over the course of the first 6 mo of lactation.

\section{METHODS}

Participants. Ninety-eight pregnant atopic women in Western Australia were recruited for a double-blind, randomized, controlled study to determine whether supplementation with n-3 LCPUFAs during pregnancy could modulate neonatal immune response with a view to preventing the development of infant allergies. Women were recruited between 16 and 20 wk of gestation from antenatal clinics of private obstetricians in Perth, Western Australia. Only mothers who delivered after 36 wk of gestation were included. All women had a history of physician-diagnosed allergic rhinitis and/or asthma and one or more positive skin prick tests to common allergens (house dust mite, grass pollens, moulds, cat, dog, and cockroach extracts) (Hollister Stier Laboratories, Spokane, WA). Women were ineligible for the study if they smoked, had other medical problems, or their normal dietary intake exceeded two meals of fish per week.

Study design and intervention. The groups were block randomized according to parity (no previous term childbirth versus one or more), prepregnancy body mass index (BMI), age, and maternal allergy (allergic rhinitis or asthma). Women in the fish oil group $(n=52)$ received four $(1-\mathrm{g})$ fish oil capsules per day (Ocean Nutrition, Halifax, Nova Scotia, Canada), comprising a total of $3.7 \mathrm{~g}$ of $\mathrm{n}-3$ LCPUFAs with $56.0 \%$ as docosahexaenoic acid

Abbreviations: EPA, eicosapentaenoic acid; DHA, docosahexaenoic acid; DPA, docosapentaenoic acid; GMDS, Griffiths Mental Development Scales; LCPUFAs, long-chain polyunsaturated fatty acids 
(DHA) (22:6n-3) and $27.7 \%$ as eicosapentaenoic acid (EPA) (20:5n-3). The control group $(n=46)$ received four $(1-\mathrm{g})$ capsules of olive oil per day (containing 66.6\% n-9 oleic acid and less than 1\% n-3 LCPUFAs) (Pan Laboratories, Moorebank, NSW, Australia). The study was approved by the Ethics Committees at St. John of God Hospital and Princess Margaret Hospital, and all women gave informed consent.

Fatty acid analysis in breast milk. Breast milk samples were collected $3 \mathrm{~d}$, $6 \mathrm{wk}$, and 6 mo postpartum, either by manual expression or breast pump, frozen immediately, and stored at $-80^{\circ} \mathrm{C}$ until analysis. Milk was thawed, and fatty acid analysis performed on whole milk samples as previously described (7). Lipids were extracted with chloroform: methanol (2:1) using butylated hydroxyanisole as an antioxidant. Fatty acid methyl esters from milk lipid extracts were prepared by acid transmethylation using $1 \% \mathrm{H}_{2} \mathrm{SO}_{4}$ in methanol. Fatty acid methyl esters were separated using a gas chromatograph (Shimadzu GC-14A, Shimadzu Corporation, Kyoto, Japan) equipped with a 50-m capillary column (0.3-mm internal diameter) coated with BPX-70 (0.25-mm film thickness; SGE Pty Ltd., Ringwood, Australia) using a modification of a previously published method (8). Each sample $(3 \mu \mathrm{L})$ was injected on to the column using an automatic injector (Shimadzu AOC-14, Shimadzu Corporation) at a split ratio of 30:1. The injector temperature was set at $250^{\circ} \mathrm{C}$ and the detector (flame ionization) temperature at $300^{\circ} \mathrm{C}$. The initial oven temperature was $140^{\circ} \mathrm{C}$ and was programmed to increase to $230^{\circ} \mathrm{C}$ at $5^{\circ} \mathrm{C} / \mathrm{min}$. Helium was used as the carrier gas at a velocity of $4 \mathrm{~mL} / \mathrm{min}$. Fatty acids were identified based on retention time to authentic lipid standards (Nu-Chek-Prep Inc., Elysian, MN) and expressed as mean percentage and SD of the total fatty acids measured.

Fatty acid analysis in erythrocytes. Blood samples were collected from the women at $36 \mathrm{wk}$ of gestation and $6 \mathrm{wk}$ postpartum and from the infants at $1 \mathrm{y}$ of age into heparinized Roswell Park Memorial Institute (RPMI, Life Technology, UK) tissue culture medium and centrifuged at $500 \times g$ for 30 min. Erythrocytes were isolated from below a Lymphoprep (Nycomed Pharmacia, Norway) gradient interface and washed in phosphate buffered saline. Fatty acid composition analyses were carried out as previously described (9). Briefly, total lipids were extracted with chloroform:methanol (2:1) and phospholipids isolated by thin-layer chromatography. Fatty acid methyl esters were prepared by treatment of phospholipid extracts with $4 \% \mathrm{H}_{2} \mathrm{SO}_{4}$ in methanol at $90^{\circ} \mathrm{C}$ for $20 \mathrm{~min}$ and analyzed by gas liquid chromatography using a Hewlett-Packard model 5980A gas chromatograph. The column was a BPX70 $(25 \times 0.32 \mathrm{~mm}, 0.25-\mu \mathrm{m}$ film thickness) (SGE, Ringwood, Victoria, Australia) with a temperature programmed from 150 to $210^{\circ} \mathrm{C}$ at $41^{\circ} \mathrm{C} /$ min and using N2 as the carrier gas at a split ratio of 30:1. Fatty acids were identified by comparison with a known standard mixture and expressed as a percentage of the weight of the total fatty acids measured (C14-C22).

Clinical outcomes. Infants were clinically evaluated at $12 \mathrm{mo}$ of age by a pediatrician/allergist (S.L.P.), who remained blinded to the intervention. The children were examined for evidence of allergy including asthma, atopic dermatitis, food allergy, and sensitization, as previously described and reported (4).

At $2.5 \mathrm{y}$ of age, children were evaluated by a psychologist who remained blinded to the intervention. Height, weight, and head circumference were measured, and the children were tested for development [Griffiths Mental Development Scales (GMDS)] (10), receptive language (Peabody Picture Vocabulary Test IIIA) (11), and behavior (Child Behavior Checklist 1-5 y) as previously described and reported (5). These tests were chosen because they have valid and reliable psychometric properties for assessing children aged $2 \mathrm{y}$. The GMDS comprise six subscales of development (locomotor, personal social, speech and hearing, eye and hand coordination, performance, and practical reasoning). One quotient was calculated for each subscale, as well as a general quotient that was derived as the mean of the subscale quotients.

Data analysis. Differences between the groups were determined by independent $t$ test. Differences in categorical variables were determined by logistic regression. The effect of treatment and time (group $\mathrm{x}$ time interaction) on fatty acid composition was examined using general linear model (GLM) repeated measures. A paired $t$ test was used for within-group analyses when a significant group $\mathrm{x}$ time interaction was shown. The two groups were also combined to determine associations between individual fatty acid proportions in breast milk and erythrocytes using Pearson's correlation. All statistical analyses were performed using SPSS software (Version 13 for Macintosh). A $p$ value $<0.05$ was considered statistically significant for the analysis of clinical outcome variables; $p<0.01$ was considered statistically significant for all fatty acid analyses because of the number of comparisons made.

\section{RESULTS}

Population characteristics. Of the 83 mothers who completed the original study (4), 73 women provided breast milk samples at one or more time points. Fifty mothers were still breast-feeding 6 mo postpartum $(n=23$ in the fish oil group and $n=27$ in the olive oil group). The infant formulas used after cessation of breast-feeding was not supplemented with LCPUFAs. There were no significant differences in the characteristics of these groups for any of the parameters examined including maternal age, prepregnancy BMI, maternal allergic status, parity, gestational length, or neonatal anthropometric measurements (data not shown). Other information about this population has been previously reported (4).

Longitudinal analysis of breast milk fatty acid composition. The composition of fatty acids in breast milk at $3 \mathrm{~d}(n=73), 6$ wk $(n=67)$, and 6 mo $(n=50)$ following delivery (and the cessation of supplementation) is shown in Table 1. Colostrum collected from the fish oil group ( $3 \mathrm{~d}$ after delivery) had significantly higher proportions of n-3 LCPUFAs [DHA, EPA, and docosapentaenoic acid (DPA) (22:5n-3)] compared with the control group ( $p<0.001$ for all measures).

The proportions of DHA and DPA decreased significantly in both groups over the first 6 wk of lactation $(p<0.001)$. However, over the three time points, the decrease was greater in the fish oil group compared with the control group (group $\times$ time interactions: $p<0.001$ ) (Table 1). Despite this, DHA $(p<0.001)$ (but not DPA) remained significantly higher in breast milk collected from the fish oil group at $6 \mathrm{wk}$. EPA also decreased significantly more in the fish oil group over the three time points $(p=0.007)$ than the control group and particularly in the first $6 \mathrm{wk}(p<0.001)$. In the control group, EPA actually showed a proportional increase between the sample collected on d 3 and the one collected 6 wk postpartum. Despite this, EPA levels remained higher in the fish oil group 6 wk postpartum. By 6 mo, there were no differences between the groups for any of the fatty acids measured.

Effects on omega-6 (n-6) LCPUFAs were less consistent. 20:3n-6 was significantly lower in the d-3 breast milk from the fish oil group $(p<0.001)$ compared with the control group. Arachidonic acid (AA) (20:4n-6) was also decreased in the fish oil group in the $\mathrm{d}-3$ sample only $(p=0.045)$.

The ratio of $n-3: n-6$ in breast milk was significantly higher in colostrum $(3 \mathrm{~d})$ from the fish oil group $(p<0.001)$ and decreased more significantly over the first $6 \mathrm{wk}$ of lactation in the fish oil group $(p<0.001)$ compared with the control group $(p=0.013)$, as shown in Figure 1, and was no longer significantly higher than the control group by $6 \mathrm{wk}$.

Association between fatty acids in maternal erythrocytes and breast milk. Women supplemented with fish oil had significantly higher n-3 LCPUFA composition (as a percentage of total fatty acids) in erythrocyte membranes at $36 \mathrm{wk}$ of gestation (total n-3 LCPUFAs: $22.8 \pm 0.4 \%$ for the fish oil and $16.2 \pm 0.4 \%$ for the placebo group; $p<0.001$ ) and $6 \mathrm{wk}$ postpartum (total n-3 LCPUFAs: $19.4 \pm 0.3 \%$ for the fish oil and $15.4 \pm 0.3 \%$ for the placebo group; $p<0.001$ ), as previously reported (12). When data from both groups were combined in a whole population analysis $(n=73)$, maternal n-3 LCPUFA levels at 36 wk of gestation were strong predictors of breast milk LCPUFA composition for the first $6 \mathrm{wk}$ of lactation, as shown in Table 2. In particular, maternal erythrocyte DHA and EPA at 36 wk of gestation correlated with 
Table 1. Breast milk fatty acids $3 d, 6 \mathrm{wk}$, and 6 mo postpartum in women supplemented with fish oil or olive oil (control) in pregnancy

\begin{tabular}{|c|c|c|c|c|c|}
\hline Fatty acids & Group & \multicolumn{3}{|c|}{$\%$ of total fatty acids } & $\begin{array}{c}\text { Group } \times \text { time } \\
p\end{array}$ \\
\hline $18: \ln 9$ & Fish oil & $33.32 \pm 4.1$ & $33.84 \pm 3.1$ & $34.37 \pm 3.9$ & 0.155 \\
\hline \multirow[t]{2}{*}{$18: 2 \mathrm{n} 6$} & Fish oil & $8.84 \pm 2.1$ & $9.91 \pm 2.3$ & $10.81 \pm 1.9$ & 0.988 \\
\hline & Control & $8.74 \pm 1.9$ & $10.71 \pm 3.4$ & $10.61 \pm 2.7$ & \\
\hline $18: 3 n 3$ & Fish oil & $0.7 \pm 0.3$ & $0.93 \pm 0.3$ & $1.16 \pm 0.5$ & 0.926 \\
\hline $20: 2 \mathrm{n} 6$ & Control & $0.44 \pm 0.1$ & $0.21 \pm 0.1$ & $0.16 \pm 0.0$ & \\
\hline \multirow[t]{2}{*}{$20: 3 n 6$} & Fish oil & $0.43 \pm 0.1 *$ & $0.35 \pm 0.1$ & $0.25 \pm 0.0$ & 0.039 \\
\hline & Control & $0.55 \pm 0.1$ & $0.37 \pm 0.1$ & $0.24 \pm 0.0$ & \\
\hline \multirow[t]{2}{*}{$20: 4 n 6$} & Fish oil & $0.55 \pm 0.1$ & $0.35 \pm 0.1$ & $0.36 \pm 0.1$ & 0.098 \\
\hline & Control & $0.61 \pm 0.1$ & $0.38 \pm 0.1$ & $0.37 \pm 0.1$ & \\
\hline $20: 5 \mathrm{n} 3$ & Fish oil & $0.16 \pm 0.1 *$ & $0.09 \pm 0.0 \dagger \dagger$ & $0.10 \pm 0.1$ & 0.007 \\
\hline $22: 6 n 3$ & Control & $0.5 \pm 0.2$ & $0.25^{\dagger} \pm 0.1 \ddagger$ & $0.34 \pm 0.4$ & \\
\hline
\end{tabular}

Data are based on breast milk collected at $3 \mathrm{~d}$ (fish oil, $n=33$; control, $n=40$ ), 6 wk (fish oil, $n=30$; control, $n=37$ ), and 6 mo (fish oil, $n=23$; control, $n=27$ ) postpartum. Values for fatty acids are expressed as a percentage of total fatty acids (mean $\pm \mathrm{SD}$ ).

$* p<0.001, \dagger p<0.01$; between-group differences.

$\$$ Within-group changes between the time points (paired $t$ test, $p<0.01$ ) if there is a subsequent difference from the d-3 level.

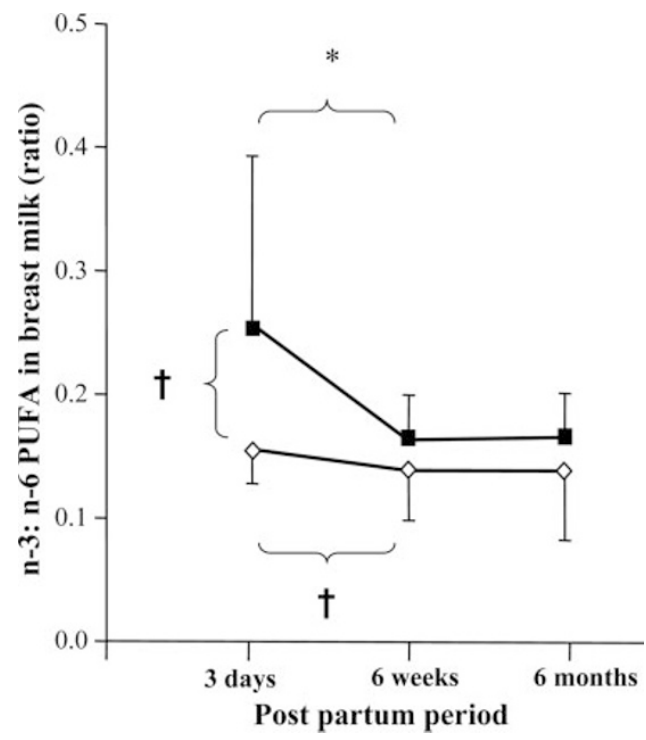

Figure 1. The ratio of n-3:n-6 in breast milk collected from women, supplemented during pregnancy with fish oil $(\mathbf{\square})$ or olive oil $(\diamond), 3 \mathrm{~d}, 6 \mathrm{wk}$, and 6 mo postpartum. A significant difference between the groups was seen for the 3-d sample $(\dagger p<0.001)$. Significance levels are also shown for within-group changes over time ( $\uparrow p<0.001$ and $* p=0.013$ ), as determined by paired $t$ tests), and denote significant changes in both groups between $3 \mathrm{~d}$ and $6 \mathrm{wk}$ postpartum.

DHA and EPA, respectively, in breast milk collected at $3 \mathrm{~d}$ $(p<0.001)$ and 6 wk postpartum $(p<0.001)$ (Table 2$)$. Maternal erythrocyte DPA was not correlated with any breast milk fatty acids; however, breast milk DPA (3 d and 6 wk postpartum) was correlated with erythrocyte EPA and DHA at 36 wk of gestation $(p<0.01)$. There were no significant correlations at 6 mo postpartum (data not shown). Maternal
Table 2. Correlation coefficients between LC-PUFAs in erythrocyte membranes collected from women at 36 wk of gestation and LC-PUFAs in breast milk collected $3 d$ and 6 wk postpartum ( $p p$ )

\begin{tabular}{cccc}
\multicolumn{4}{c}{ postpartum $(\mathrm{pp})$} \\
& \multicolumn{3}{c}{ Maternal fatty acids at 36 wk of gestation } \\
\cline { 2 - 4 } & EPA & DPA & DHA \\
\hline Breast milk 3 & & & \\
pp $(\mathrm{n}=72)$ & -0.265 & -0.004 & -0.186 \\
AA & $0.848^{*}$ & -0.029 & $0.800^{*}$ \\
EPA & $0.618^{*}$ & 0.158 & $0.639^{*}$ \\
DPA & $0.754^{*}$ & 0.122 & $0.782^{*}$ \\
DHA & & & \\
Breast milk 6 wk & & & \\
pp (n = 66) & 0.273 & 0.006 & -0.13 \\
AA & $0.455^{*}$ & 0.11 & $0.432^{*}$ \\
EPA & $0.332 \dagger$ & 0.133 & $0.325 \dagger$ \\
DPA & $0.617 \dagger$ & 0.105 & $0.577^{*}$ \\
DHA &
\end{tabular}

Significant Pearson's correlation: $* p<0.001 ; \dagger p<0.01$.

erythrocyte EPA and DHA 6 wk postpartum was strongly correlated $(p<0.001)$ with breast milk EPA and DHA collected at the same time point (Table 3).

Association between breast milk fatty acids and infant fatty acids at $1 \quad y$. Figure 2 shows the change in infant erythrocyte fatty acid composition between birth and 12 mo of age for a subgroup with available samples (29 children in each group). DHA (22:6n-3) was significantly lower $(p<0.001)$ at 12 mo in both groups. At 1 y, EPA (20:5n-3) was significantly higher $(p<0.001)$ in the control group and significantly lower $(p<0.001)$ in the fish oil group compared with birth. N-6 PUFA 20:3n-6, 20:4n-6, and 22:4n-6 composition was also significantly lower $(p<0.01)$ at 12 mo of age compared with 
Table 3. Correlation coefficients between LC-PUFAs in erythrocyte membranes collected from women $6 \mathrm{wk}$ postpartum and LC-PUFAs in breast milk collected 6 wk and 6 mo pp

\begin{tabular}{cccc}
\hline & \multicolumn{3}{c}{ Maternal fatty acids 6 wk pp } \\
\cline { 2 - 4 } & EPA & DPA & DHA \\
\hline Breast milk 6 wk & & & \\
pp $(n=64)$ & -0.1 & 0.142 & -0.109 \\
AA & $0.620^{*}$ & 0.135 & $0.438^{*}$ \\
EPA & $0.40 \dagger^{\dagger}$ & $0.259 \dagger$ & $0.348^{\dagger}$ \\
DPA & $0.568^{*}$ & 0.217 & $0.598^{*}$ \\
DHA & & & \\
Breast milk 6 mo & & & \\
pp $(n=47)$ & 0.112 & 0.181 & 0.041 \\
AA & 0.331 & 0.149 & 0.267 \\
EPA & 0.095 & 0.117 & 0.06 \\
DPA & 0.155 & 0.134 & 0.181 \\
DHA &
\end{tabular}

Significant Pearson's correlation: $* p<0.001 ; \dagger p<0.01$.

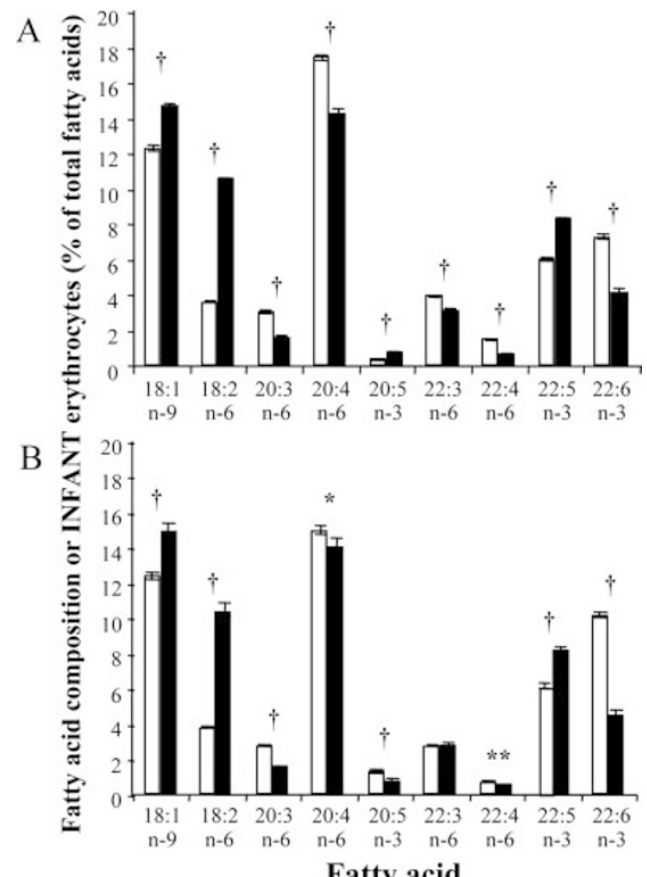

Figure 2. Comparison of relative fatty acid composition of cord blood erythrocytes $(\square)$ with peripheral blood erythrocytes $(\square)$ collected from the infants at $1 \mathrm{y}$ of age are shown for the control group $(A)$ and the fish oil group $(B)$. Values shown are the mean percentage of the total fatty acids measured ( \pm SEM) in the control group $(n=29)$ and the fish oil group $(n=29)$. Statistically significant changes determined by paired $t$ test and indicated as $* p<0.05, * * p<0.01, \dagger p<0.001$.

fatty acid composition at birth in both groups (Fig. 2). In contrast, lactic acid (18:2n-6) was significantly higher $(p<$ 0.001 ) at $1 \mathrm{y}$ compared with composition at birth in both the fish oil and control groups.

There was no significant difference in the fatty acid status of infants in the fish oil compared with the control group at $1 \mathrm{y}$ of age. However, when the groups were combined in whole population analyses, there were strong and consistent correlations between DHA status in infants at 12 mo of age and DHA in breast milk collected $3 \mathrm{~d}(n=52 ; r=0.328, p=0.018)$, 6 wk $(n=45 ; r=0.510, p<0.001)$ and 6 mo $(n=35 ; r=$
$0.506, p=0.002$ ) postpartum. It was also noted that infants who were breast-fed for at least 6 mo had higher DHA at 12 mo of age (mean, $4.97 \pm \mathrm{SD}: 1.55 \%$ total fatty acids) than those breast-fed less than 6 mo (or not at all) (mean, $3.49 \pm$ SD: $0.86 \%$ total fatty acids, $p<0.001)$. After adjusting for differences in feeding method in a linear regression model, fish oil supplementation during pregnancy did not significantly affect DHA status at 1 y of age $[\beta$ coefficient: -0.46 ; $95 \%$ confidence interval $(\mathrm{CI}):-1.15$ to $0.22, p=0.283$ ]. This suggests that fatty acid status at $1 \mathrm{y}$ of age was more a function of postnatal intake (including breast milk) than of fatty acid levels in pregnancy.

Association between breast milk fatty acids and clinical outcomes in the infants. We previously reported the effects of the fish oil supplementation in this population on infant growth (5), neurodevelopment (5), and allergic outcomes (4). Here we examine the relationship between breast milk n-3 LCPUFA status and these previously noted outcomes.

First, we examined breast milk composition in relation to the previously noted effects of fish oil on allergy outcomes. Although the original study was not designed to assess effects on clinical allergy, we previously reported that infants of fish oil-supplemented mothers were less likely to be sensitized to egg at $1 \mathrm{y}$ [odds ratio $(\mathrm{OR})=0.34 ; 95 \% \mathrm{CI}: 0.11-1.02 ; p=$ 0.055] (4). Here, we also found that infants still breast-fed at 6 mo of age were more likely to be sensitized to egg in the first year of life $(\mathrm{OR}=3.43 ; 95 \% \mathrm{CI}: 1.01-11.66 ; p=0.048)$. Since breast milk is a continued source of DHA in the postnatal period, we performed logistic regression between individual fatty acids in breast milk and sensitization; however, there were no significant associations. Furthermore, in a logistic regression model, fish oil supplementation and breastfeeding were found to both have independent effects.

Second, we examined breast milk composition in relationship to the previously noted effects of fish oil on neurodevelopmental outcomes, including improved hand-eye coordination in the babies of the fish oil group at $2.5 \mathrm{y}$ of age (5). As shown in Table 4, we found that both EPA and DHA content of breast milk was positively correlated with nearly all the GMDS subscales of development including hand and eye coordination (EPA: $r=0.405, p=0.001$; DHA: $r=0.315$, $p=0.016)$. High AA in breast milk collected 6 mo postpartum was associated with reduced vocabulary skills as measured by the average length of phrase used $(r=-0.533, p=$ $0.002)$ and the number of words used $(r=-0.371, p=$ 0.028).

Finally, we examined the relationship between breast milk n-3 LCPUFA status and infant growth parameters at $2.5 \mathrm{y}$ of age. There were no differences in growth (height, weight, and head circumference) between the fish oil and the control groups, before and after adjusting for breast-feeding (data not shown).

\section{DISCUSSION}

Our existing study population provided a novel opportunity to examine the effects of supplementation in pregnancy (but not lactation) on breast milk over the longer term. Many 
Table 4. Correlation coefficients between scores for cognitive outcomes in children at 2.5 years of age and LC-PUFAs in breast milk collected from their mothers $3 \mathrm{~d}$ and $6 \mathrm{wk} p$

\begin{tabular}{|c|c|c|c|c|c|c|}
\hline \multirow{2}{*}{$\begin{array}{l}\text { Cognitive outcomes from } \\
\text { GMDS }\end{array}$} & \multicolumn{3}{|c|}{ Breast milk fatty acids $3 \mathrm{~d}$ pp $(n=64)$} & \multicolumn{3}{|c|}{ Breast milk fatty acids 6 mo pp $(n=58)$} \\
\hline & EPA & DPA & DHA & EPA & DPA & DHA \\
\hline Locomotor & $0.273 *$ & 0.172 & $0.256^{*}$ & 0.155 & 0.173 & 0.231 \\
\hline Social & 0.244 & 0.237 & $0.271 *$ & 0.141 & 0.094 & 0.25 \\
\hline Speech and hearing & $0.288^{*}$ & 0.239 & $0.249 *$ & 0.193 & 0.162 & 0.21 \\
\hline Eye and hand coordination & $0.405 \dagger$ & 0.226 & $0.318 *$ & $0.315^{*}$ & 0.211 & 0.237 \\
\hline Performance & $0.324 *$ & $0.253^{*}$ & $0.277 *$ & $0.310 *$ & $0.278 *$ & 0.252 \\
\hline Practical reasoning & 0.179 & 0.062 & 0.097 & $0.276^{*}$ & 0.102 & 0.146 \\
\hline General quotient & $0.344 \dagger$ & 0.238 & $0.297 *$ & $0.293 *$ & 0.219 & $0.273 *$ \\
\hline
\end{tabular}

Significant Pearson's correlation: $* p<0.05, \dagger p<0.01$.

previous studies were only short term (13) or used fish oil during lactation [with (14) or without (15) supplementation during pregnancy as well]. To our knowledge, this is the first study to document the effects of high-dose fish oil supplementation during pregnancy on longitudinal changes in breast milk fatty acid composition over the first 6 mo of lactation. The most significant effect was on breast milk n-3 LCPUFAs, particularly DHA and EPA levels, which remained significantly higher over the first $6 \mathrm{wk}$ of lactation in the women supplemented with fish oil compared with controls. The ratio of $n-3: n-6$ was also significantly higher in this period in the fish oil group. With growing recognition of the importance of n-3 LCPUFAs, particularly DHA, for early development, these observations are highly relevant to recent strategies to optimize early n-3 LCPUFA status in this early period. Although these infants already had significantly higher $n-3$ LCPUFA levels at birth as a result of maternal supplementation in pregnancy [as previously reported (12)], our findings here confirm additional postnatal nutritional benefits of improved maternal n-3 LCPUFA status for breast-fed infants. As anticipated, the increases in breast milk n-3 LCPUFA content were greatest in the first months of lactation and were seen without mothers continuing supplementation in the postnatal period. Our findings contrast with those of Boris et al. (13) who noted that fish oil supplementation limited to pregnancy was not as effective as supplementation during both pregnancy and lactation and did not significantly increase n-3 LCPUFA content in breast milk compared with a control group.

As also anticipated, we have confirmed that maternal n-3 LCPUFA status in late pregnancy is strongly correlated with $\mathrm{n}-3$ LCPUFAs in breast milk for the first 6 wk of lactation. These relationships are particularly strong for both EPA and DHA. These observations indicate that maternal diet during pregnancy is an important source of these fatty acids in early lactation and reinforces the additional benefits of supplementation in pregnancy on postnatal DHA status, for both mother and infant. It is important to note that the effects observed in this study were achieved using higher doses of fish oil (3.7 g of $n-3$ LCPUFA per day in pregnancy) than are sometimes used in pregnancy and in most previous studies of supplementation during pregnancy. Notably, the levels of 18:1 fatty acids were similar between the groups, even though the control group received this (oleic acid 18:1). This suggests selective deposition and mobilization of fatty acids during pregnancy and lactation, favoring LCPUFAs. The relationship between breast milk fatty acid at $6 \mathrm{wk}$ and maternal (erythrocyte) fatty acid was just as significant for erythrocytes analyzed at $6 \mathrm{wk}$ as it was for erythrocytes analyzed at term (Tables 2 and 3), indicating that maternal fatty acid status at delivery is a good predictor of subsequent breast milk composition many weeks later. These relationships also suggest that the slight decline in maternal n-3 LCPUFA stores after gestation could be the result (at least in part) of diversion of these fatty acids into breast milk production.

We have previously reported (in this population) that infants in the fish oil group were less likely to develop allergic sensitization to egg allergen (4) and that they also had improved neurodevelopment outcomes [notably in hand-eye coordination at $2.5 \mathrm{y}(5)]$ compared with the control group. Importantly, there were no adverse effects of this selective supplementation on growth (or development). Although it is difficult to separate the direct antenatal effects of supplementation on clinical outcomes from additional postnatal effects of breast milk, we have shown that effects on breast milk have a significant independent effect on fatty acid status at $1 \mathrm{y}$. Specifically, although there was no differences in $1 \mathrm{y}$ fatty acid status of infants in the fish oil group compared with the controls (i.e. no relationship with antenatal supplementation per se), it is of note that there were strong and consistent correlations between breast milk fatty acid composition and infant fatty acid status at $1 \mathrm{y}$. Significantly, this suggests that postnatal fatty acid intake is a stronger indicator of fatty acid status at $1 \mathrm{y}$ of age than whether the infants' mothers were supplemented in pregnancy. In particular, DHA composition in breast milk at $3 \mathrm{~d}, 6 \mathrm{wk}$, and 6 mo postpartum was significantly correlated with infant DHA at $1 \mathrm{y}$ in a combined population analysis. Furthermore, infants who were exclusively breast-fed for at least 6 mo had higher DHA and total n-3 LC-PUFAs at 12 mo of age than those breast-fed less than $6 \mathrm{mo}$ or not at all. These findings reinforce the importance of DHA levels in breast milk for maintaining subsequent infant DHA status. Although more LCPUFAs are deposited in the brain during the last trimester compared with postnatal life, there is evidence that deposition continues beyond term (16). Neurologic development continues in the first years of life, and some studies suggest that LCPUFA supplementation in the postnatal period can improve functional outcomes (17), suggesting that postnatal deposition is also important. It is therefore possible that the clinical benefits of fish oil supplementation in pregnancy will also be mediated (in part) by 
additional postnatal effects via breast milk. This study highlights the need to consider these additional effects in pregnancy supplementation studies. Further studies are currently under way in our group to assess the separate effects of fish oil in the postnatal period. This will help address the relative contribution of "pre" versus "post" natal LCPUFA status.

We acknowledge that daily variations in breast-milk LCPUFAs limit the ability of a single milk sample to give a precise measure of the n-3 LCPUFA intake. However, our observations here suggest that this can still be a useful measure.

In summary, we have demonstrated that fish oil supplementation during the second half of pregnancy modified breast milk LC-PUFA composition and that breast milk composition (rather than pregnancy levels) contributed to infant n-3 LCPUFA status at $1 \mathrm{y}$ of age. This suggests that in addition to improving neonatal n-3 LCPUFA status, supplementation in pregnancy has continued effects on infant fatty acid status through effects on breast milk composition. Although the importance of achieving "adequate" n-3 LCPUFA levels is well established (and highly relevant to many Western societies where n-3 LCPUFA intakes have been declining), the role of higher dose supplementation to prevent specific diseases or enhance cognitive development is not established and still requires further study.

Acknowledgments. The authors thank the mothers and babies who participated in the study.

\section{REFERENCES}

1. Meyer BJ, Mann NJ, Lewis JL, Milligan GC, Sinclair AJ, Howe PR 2003 Dietary intakes and food sources of omega- 6 and omega-3 polyunsaturated fatty acids. Lipids 38:391-398

2. Makrides M, Simmer K, Neumann M, Gibson R 1995 Changes in the polyunsaturated fatty acids of breast milk from mothers of full-term infants over $30 \mathrm{wk}$ of lactation. Am J Clin Nutr 61:1231-1233
3. Oken E, Kleinman KP, Berland WE, Simon SR, Rich-Edwards JW, Gillman MW 2003 Decline in fish consumption among pregnant women after a national mercury advisory. Obstet Gynecol 102:346-351

4. Dunstan JA, Mori TA, Barden A, Beilin LJ, Taylor AL, Holt PG, Prescott SL 2003 Fish oil supplementation in pregnancy modifies neonatal allergen-specific immune responses and clinical outcomes in infants at high risk of atopy: a randomized, controlled trial. J Allergy Clin Immunol 112:1178-1184

5. Dunstan JA, Simmer K, Dixon G, Prescott SL 2006 Cognitive assessment at $2 \frac{1}{2}$ years following fish oil supplementation in pregnancy: a randomized controlled trial. Arch Dis Child Fetal Neonatal Ed [Epub ahead of print]

6. Jensen CL, Maude M, Anderson RE, Heird WC 2000 Effect of docosahexaenoic acid supplementation of lactating women on the fatty acid composition of breast milk lipids and maternal and infant plasma phospholipids. Am J Clin Nutr 71:292S-299S

7. Dunstan JA, Roper J, Mitoulas L, Hartmann PE, Simmer K, Prescott SL 2004 The effect of supplementation with fish oil during pregnancy on breast milk immunoglobulin A, soluble CD14, cytokine levels and fatty acid composition. Clin Exp Allergy 34:1237-1242

8. Makrides M, Neumann MA, Simmer K, Gibson RA 1995 Erythrocyte fatty acids of term infants fed either breast milk, standard formula, or formula supplemented with long-chain polyunsaturates. Lipids 30:941-948

9. Mori TA, Burke V, Puddey IB, Watts GF, O'Neal DN, Best JD, Beilin LJ 2000 Purified eicosapentaenoic and docosahexaenoic acids have differential effects on serum lipids and lipoproteins, LDL particle size, glucose, and insulin in mildly hyperlipidemic men. Am J Clin Nutr 71:1085-1094

10. Griffiths R 1970 The abilities of young children. A comprehensive system of mental measurement for the first eight years of life. London, Child Development Research Centre, 1970. Child Development Research Centre, London

11. Dunn L, Dunn L 1997 Examiners manual for the Peabody Picture Vocabulary Test. American Guidance Service, Circle Pines, MN

12. Dunstan JA, Mori TA, Barden A, Beilin LJ, Holt PG, Calder PC, Taylor AL, Prescott SL 2004 Effects of n-3 polyunsaturated fatty acid supplementation in pregnancy on maternal and fetal erythrocyte fatty acid composition. Eur J Clin Nutr 58:429-437

13. Boris J, Jensen B, Salvig JD, Secher NJ, Olsen SF 2004 A randomized controlled trial of the effect of fish oil supplementation in late pregnancy and early lactation on the n-3 fatty acid content in human breast milk. Lipids 39:1191-1196

14. Helland IB, Saugstad OD, Saarem K, Van Houwelingen AC, Nylander G, Drevon CA 2006 Supplementation of n-3 fatty acids during pregnancy and lactation reduces maternal plasma lipid levels and provides DHA to the infants. J Matern Fetal Neonatal Med 19:397-406

15. Lauritzen L, Jørgensen MH, Mikkelsen TB, Skovgaard M, Straarup EM, Olsen SF, Høy CE, Michaelsen KF 2004 Maternal fish oil supplementation in lactation: effect on visual acuity and n-3 fatty acid content of infant erythrocytes. Lipids 39:195-206

16. Makrides M, Neumann MA, Byard RW, Simmer K, Gibson RA 1994 Fatty acid composition of brain, retina, and erythrocytes in breast- and formula-fed infants. Am J Clin Nutr 60:189-194

17. Uauy R, Hoffman DR, Mena P, Llanos A, Birch EE 2003 Term infant studies of DHA and ARA supplementation on neurodevelopment: results of randomized controlled trials. J Pediatr 143:S17-S25 